\title{
Caracterización morfométrica y determinación de índices zoométricos de un hato de ganado criollo Blanco Orejinegro (BON) puro, en Pacho
}

\section{(Cundinamarca)}

\section{Characterization and identification of indices morphometric zoometric of a} pure White Orejinegro (BON) creole cattle herd, in Pacho (Cundinamarca)

\author{
Rojas Jiménez Jenny Soad ${ }^{1}$; Casas Pulido Mónica del Pilar ${ }^{1} ; \mathrm{y}$ \\ Martínez Correal Germán² \\ ${ }^{1}$ Médico Veterinario Zootecnista y ${ }^{2}$ Médico Veterinario, MSc, PhD \\ asocriollo@hotmail.com
}

Recibido 17 de Enero 2014, Aceptado 30 de Abril 2014

\section{RESUMEN}

Esta investigación fue realizada en Pacho, Cundinamarca, Colombia, donde se evaluaron vacas de la raza criolla Blanco Orejinegro (BON), tomando un total de 18 medidas Morfométricas por animal; con la ayuda de algunos materiales tales como cinta métrica y bastón zoométrico. Estas medidas permitieron la elaboración de una matriz de correlaciones para definir si estos animales responden a un modelo armónico; determinando así la "Caracterización Morfométrica de la Raza". Según el análisis de correlaciones, se encontró que en el $88,23 \%$ de los casos existe una correlación positiva entre las variables. Por lo que se define que estos animales de raza BON, poseen un alto grado de armonicidad en su modelo morfoestructural.

Palabras clave: Criollo, Blanco Orejinegro, morfometría, modelo armónico, índice zoométrico.

\section{ABSTRACT}

This research was conducted in Pacho, Cundinamarca, Colombia, where cows of the creole breed Blanco Orejinegro were evaluated with the help of a metric tape 
and zoometric gad. These measures permitted the elaboration of a correlation matrix defining that these animals correspond to a harmonic model. This way, we characterized the morphometrics characteristics of the BON breed. According to the correlation analysis, this study or the present work found that in the $88,23 \%$ of de cases exist a positive correlation between the variables. Therefore, it is defined that these animals of the BON breed have a high degree of harmonicity in their morphostructural model.

Keywords: Creole, Blanco Orejinegro, morfometric, harmonic model, index morphometric.

\section{INTRODUCCIÓN}

Las razas criollas colombianas; Blanco Orejinegro, Casanareño, Costeño con cuernos, Romosinuano, Hartón del Valle, Chino Santandereano, Velásquez y Lucerna, se originaron del ganado introducido por los conquistadores españoles en el siglo XV y hasta comienzos del siglo XX fueron la base genética de todos los sistemas de producción bovina, especialmente en el trópico bajo colombiano. Desde su introducción, fueron sometidas a un largo proceso de selección natural que les permitió adquirir características adaptativas de gran importancia económica, tales como: eficiencia reproductiva; tolerancia a enfermedades y parásitos; habilidad para soportar condiciones extremas de temperatura y humedad, y para utilizar forrajes fibrosos; constituyéndose así en el mayor patrimonio biológico y económico para la provisión de alimentos (carne, leche), pieles y trabajo en los diferentes sistemas de producción de la variada geografía del trópico colombiano (Martínez, 1995; Valderrama, 2003).

Husu, (2004) afirma que si existe una completa armonía entre el animal y su ambiente, éste progresará y será un productor eficiente, si se puede entender las particularidades de un medio ambiente, será posible seleccionar aquellos animales que en dicho ambiente están mejor adaptados. El medio ambiente es el factor decisivo determinante del tamaño y función de los animales, ya que estos poseen 
una relación directa con la disponibilidad de alimentos. El tamaño corporal es otro aspecto importante al seleccionar el genotipo, cuando los recursos alimenticios son limitados (Warris, 2004).

Por esta razón es importante llevar a cabo trabajos de investigación en donde se oriente al criador sobre el tipo de animal que tenga bajos requerimientos de mantenimiento, buen potencial de producción, altas tasas de reproducción para que valga la pena mantenerlas durante los tiempos críticos de sequía; y por último, que sean animales que sepan tomar ventaja de los periodos de abundancia de forraje (MADR, 2003).

Una de las siete razas criollas que actualmente se reconocen en el país es el Blanco Orejinegro (BON), el número de ejemplares $\mathrm{BON}$ racialmente puros que se conservan en el territorio colombiano apenas asciende a 2.866 animales (Buitrago et al., 1999). Es imperativo entonces que esta única e invaluable fuente genética sea protegida antes que desaparezca, debido a su extinción o a su disolución por el cruzamiento indiscriminado y absorbente con las razas modernas de ganados taurus o indicus, altamente eficientes en la producción de leche o carne, pero las europeas (taurus) están inadaptadas al medio tropical (Martínez, 1992; Vaca, 2002).

Uno de los mayores inconvenientes que tienen que afrontar las razas criollas es la poca difusión de sus bondades productivas. Aunque no son muchos si son importantes los estudios que se han realizado evaluando parámetros reproductivos, productivos y genéticos relacionados con el ganado criollo y especialmente con el BON. De la continuación de estos estudios y de la aplicación de sus resultados, depende que se ofrezcan a los ganaderos criadores de ganado criollo puro y comercial (BON) ejemplares seleccionados que puedan ser utilizados en el mejoramiento de las características productivas de interés económico (López et al., 2001). 
El ganado Blanco Orejinegro ofrece condiciones fisiológicas admirables para adaptarse a terrenos pobres y quebrados de nuestro medio, sobrevivir y reproducirse hasta una edad avanzada. Ha sido manejado durante muchos años solo con fines de conservación y multiplicación, sin parámetros definidos de selección, pero debido a los limitantes tecnológicos las tasas de natalidad no sobrepasan el $50 \%$, la mortalidad en jóvenes es cercana al $10 \%$ y superior al $2 \%$ en adultos; con los anteriores parámetros de reproducción y con los reducidos índices de crecimiento, inferiores a 300 gramos/día en el período predestete, el peso a esta edad (9 meses) es de escasos $136 \mathrm{~kg}$; es decir que la producción de kilos por cada vaca expuesta a toro en el hato, es inferior a $60 \mathrm{~kg} / \mathrm{vaca} / \mathrm{año}$ (Martínez, 1992; López et al., 2001).

Por lo tanto, el presente trabajo ha pretendido estudiar el uso de ciertas medidas bovinométricas que permitan no solamente establecer el biotipo de animal adecuado a nuestro sistema de producción, sino predecir las características productivas de los animales con esos biotipos, obteniendo así la uniformidad de la raza.

\section{MATERIALES Y MÉTODOS}

El proyecto se realizó en la hacienda, El Palmar de la Macorina, ubicada en la vereda Bermejal, en el municipio de Pacho, departamento de Cundinamarca, Colombia, que cuenta con una altura máxima de 1600 m.s.n.m., temperatura de $23{ }^{\circ} \mathrm{C}$ y Humedad relativa del $85 \%$ promedio anual. Los animales que se evaluaron fueron ejemplares hembras Blanco Orejinegro, vacías y mayores de 3 años.

Se tomaron 18 medidas morfométricas por animal, y se hallaron los correspondientes índices zoométricos, para lo cual la sujeción se realizó en brete manteniendo los animales en una superficie lisa, plana y nivelada, con las cuatro patas formando un rectángulo y la cabeza gacha, de manera que la nuca y la cruz 
quedarán de la misma altura. Las medidas de diámetros y longitudes, fueron tomadas con bastón zoométrico y los perímetros con cinta métrica convencional.

Los datos fueron sometidos a análisis de correlación lineal simple ( $r$ ) con el fin de determinar el grado de asociación entre las variables morfométricas como: alzada, mediciones bovinométricas, perímetro toráxico de caña, también se estableció la armonicidad del modelo morfométrico incluyendo análisis de índices morfométricos que se clasifican en raciales y funcionales utilizando el programa Statistical Analysis System (SAS), (1995).

\section{RESULTADOS Y DISCUSIÓN}

\section{Medidas Morfométricas:}

Alzadas: Alzada a la cruz (AC): $122,7 \pm 4.24 \mathrm{~cm}$. Esto indica que son animales de talla media. Valores similares se encontraron en las razas Casanareño, Caqueteño, Sanmartinero y Hartón del Valle, las cuales oscilan entre 120, 140, 130 y $129 \mathrm{~cm}$., respectivamente. Alzada a la entrada de la grupa (AEG): 127,2 \pm $3.54 \mathrm{~cm}$. Indica que son animales de mayor alzada en el tren posterior que en el anterior, posiblemente para facilitar su desplazamiento en terreno montañoso. Con la similitud de las alzadas (cruz y grupa) se deduce la rectitud de la línea dorso lumbar, la cual es de excepcional importancia en las razas bovinas especializadas en la producción de carne y trabajo principalmente (Figuras 1 y 2, Tabla 1). Esta similitud de medidas en la raza BON indica que es un animal de trabajo y su selección se debería orientar a la producción de carne. Valores similares, como 135 y $127 \mathrm{~cm}$. de AEG fueron reportados por Sierra y Velásquez (2000) y Sastre (2003) en la raza Sanmartinero y Casanareño respectivamente.

Mediciones bovinométricas: Ancho de cadera $(A n C): 49,1 \pm 4.02 \mathrm{~cm}$. Longitud de grupa (LG): $51 \pm 3.3 \mathrm{~cm}$. Ancho posterior de la grupa (AG): 47,2 $\pm 3.38 \mathrm{~cm}$. Anchura Inter-isquiática (AII): $11 \pm 1,5 \mathrm{~cm}$. Ancho del Lomo (AL): 35,8 $\pm 2,6 \mathrm{~cm}$. Longitud Occipito Isquial (LOI): $184 \pm 8,8 \mathrm{~cm}$. Distancia entre Encuentros (DE): 
41,4 $\pm 3,2 \mathrm{~cm}$. Diámetro Longitudinal $(\mathrm{DL}): 147,9 \pm 8.18 \mathrm{~cm}$. Diámetro Bicostal (DB): $68,1 \pm 4.09 \mathrm{~cm}$. Diámetro Dorsoesternal (DD): 67,7 $\pm 3,17 \mathrm{~cm}$. Longitud de Cara (CR): 30,9 $\pm 2,6 \mathrm{~cm}$. Longitud de Cráneo (LC): 19,2 $\pm 1,6 \mathrm{~cm}$. Ancho de Cabeza (ACz): $49 \pm 1,3 \mathrm{~cm}$. Longitud de Cabeza (LCz): $50 \pm 2,4 \mathrm{~cm}$ (Figuras 1 y 2 , Tabla 1).

Perímetro: Perímetro Toráxico (PT): 175,2 \pm 8,9 cm. Perímetro de Caña (PC): $16,5 \pm 0,8 \mathrm{~cm}$ (Figuras 1 y 2 , Tabla 1 ).

El diámetro longitudinal, tuvo un promedio de $147.9 \pm 8.18 \mathrm{~cm}$, siendo animales más largos que otras razas criollas como por ejemplo la Casanareña, en la cual se reporta una media, en hembras, de $138.33 \mathrm{~cm}$. La mayor longitud del BON le permite ser clasificado como un animal con mayor capacidad cárnica que el Casanareño. Respecto al DD $(67,7 \pm 3,17 \mathrm{~cm})$ datos similares fueron reportados por Sastre, (2003) con diámetros bicostales de 63,3 cm. en vacas Casanareño y $64,7 \mathrm{~cm}$. de DB en vacas Caqueteñas reportados por Floriano y Trujillo (2005).

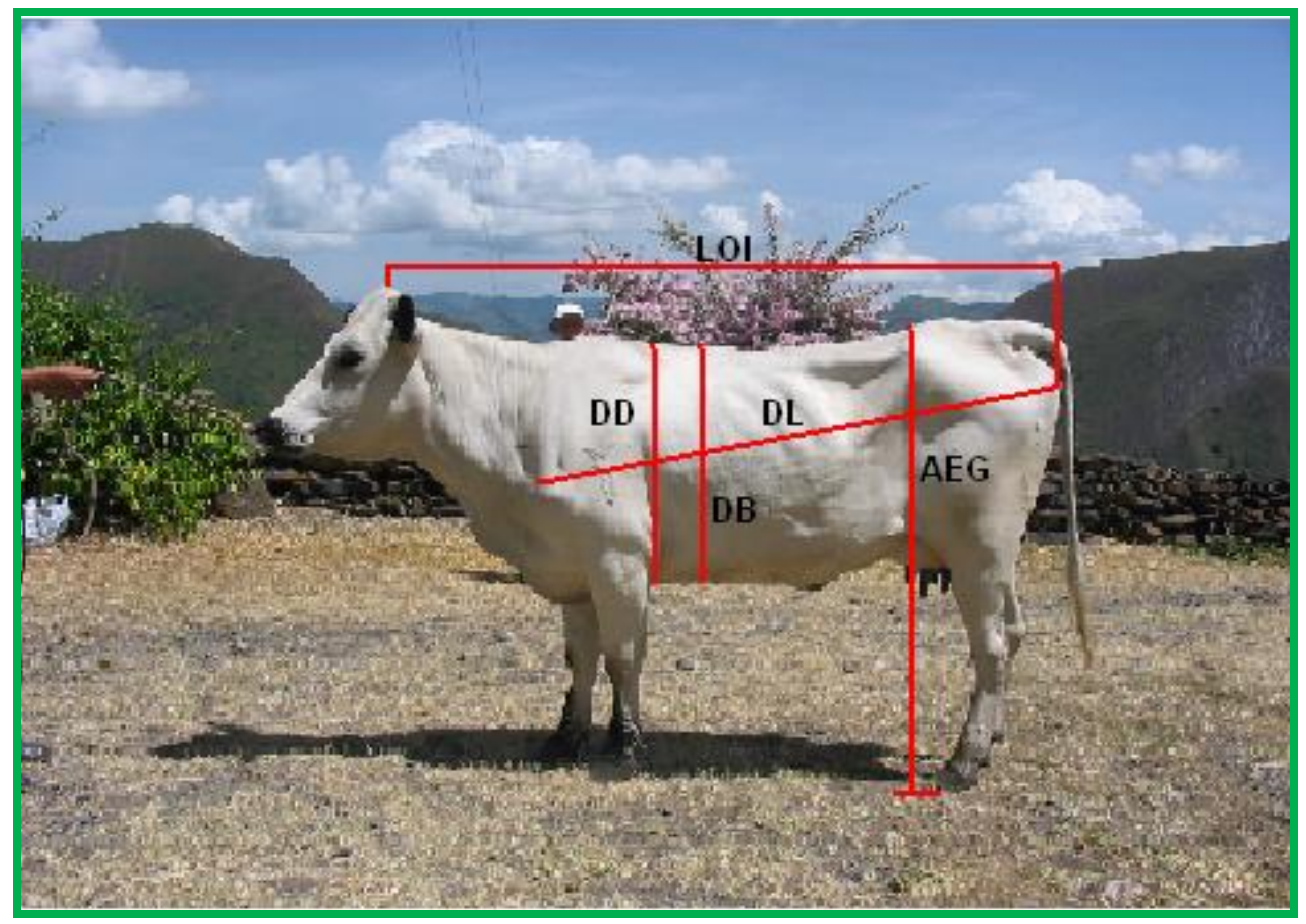

Figura 1. Algunas medidas bovinométricas: Longitud occisito- isquial (LOI), Diámetro dorso esternal (DD), Diámetro longitudinal (DL), Alzada a la entrada de la grupa (AEG), Diámetro bicostal (DB). 
Por lo anterior se deduce que las vacas BON en este estudio, tienen mayor DD y por lo tanto mayor profundidad de pecho que las vacas Casanareñas y Caqueteñas reportadas. EI DB $(68.1 \pm 4.09 \mathrm{~cm})$ presenta una diferencia de $33 \mathrm{~cm}$, con respecto a las encontradas en las vacas Casanareñas (34.47 \pm 11.67$)$ (Sastre, 2003), esta desigualdad debe estar relacionada con la variabilidad sobre el estado de carnes de los animales, así como de su capacidad reproductiva al momento del muestreo.

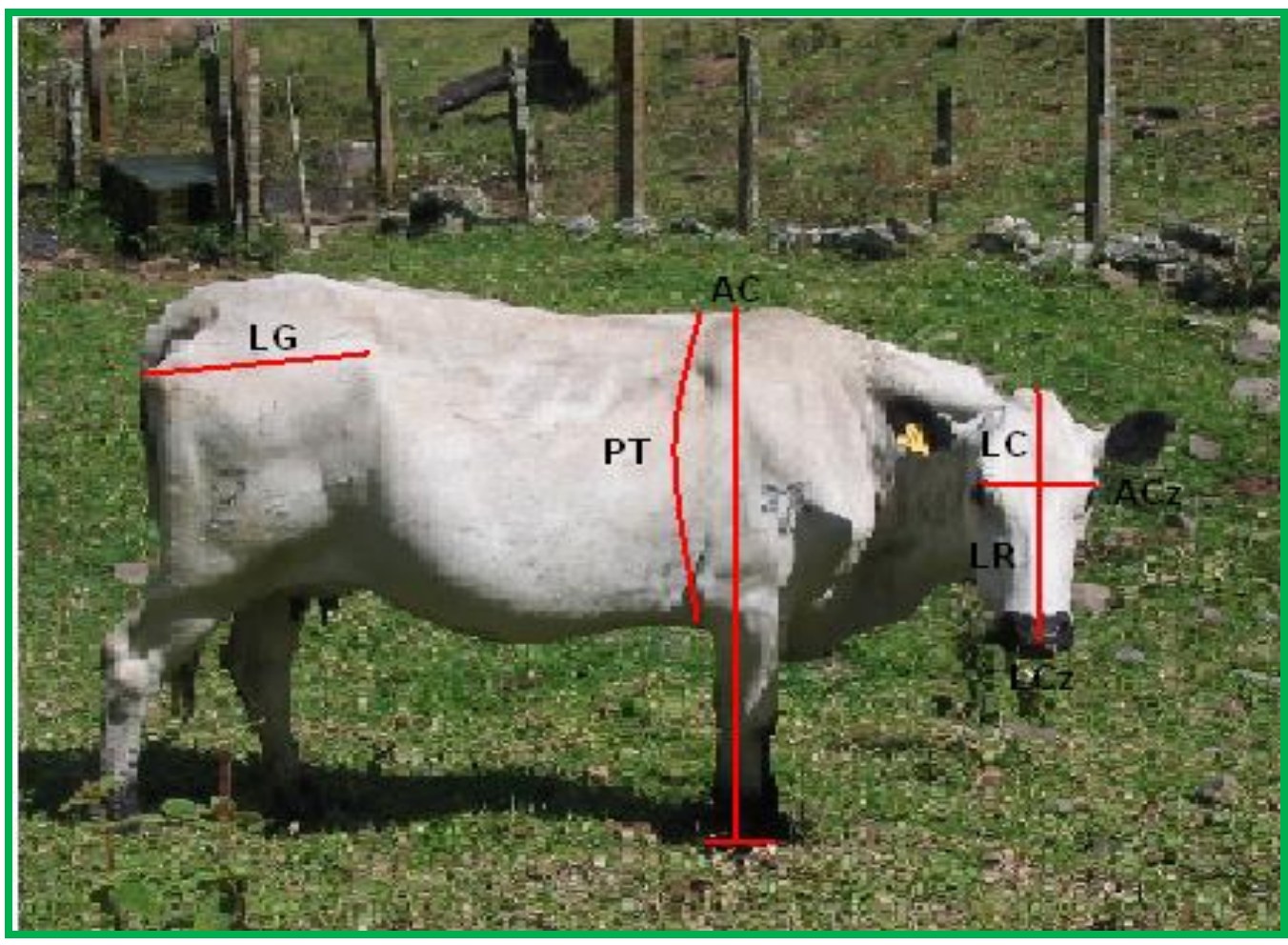

Figura 2. Algunas medidas bovinométricas: Longitud de grupa (LG), Perímetro tórax (PT), Alzada a la cruz (AC), Longitud de cráneo (LC), Longitud de cara (LR), Ancho de cabeza (ACz) y Longitud de cabeza (LCz).

\section{Estudio de la armonicidad del modelo morfométrico:}

El estudio se realizó según la metodología expuesta por Herrera et al., (2003) realizando el análisis de correlaciones simples entre las 18 variables estudiadas, se observó (Tabla 2) que en el $88.23 \%$ de los casos existe una correlación 
significativa $(p<0,05)$ entre las diferentes variables. Según estos resultados, la raza BON, presenta un grado de armonicidad alto en su modelo morfoestructural, mayor que en otras razas criollas como la Casanareña que presentó $61.7 \%$ (Sastre, 2003) y Caqueteña 77,17\% (Floriano y Trujillo, 2005) de correlaciones significativas.

Tabla 1. Valores medios, mínimos, máximos y desviación estándar de 18 medidas morfométricas tomadas en las hembras de raza Blanco Orejinegro (BON)

\begin{tabular}{|c|c|c|c|c|c|}
\hline Grupo & Variable & Media & Mínimo & Máximo & Desv. Std \\
\hline Peso (kg) & Peso & 439.042 & 280.000 & 640.000 & 68.612 \\
\hline \multirow{7}{*}{$\begin{array}{c}\text { Alzadas } \\
(\mathrm{cm})\end{array}$} & $A C$ & 122.765 & 114.000 & 133.000 & 4.243 \\
\hline & $A E G$ & 127.276 & 117.000 & 133.000 & 3.542 \\
\hline & LCz & 50.000 & 44.000 & 55.000 & 2.449 \\
\hline & LR & 30.957 & 23.000 & 36.000 & 2.653 \\
\hline & LC & 19.212 & 15.000 & 24.000 & 1.693 \\
\hline & $A C z$ & 23.340 & 21.000 & 26.000 & 1.387 \\
\hline & DB & 68.148 & 59.000 & 76.000 & 4.096 \\
\hline \multirow{9}{*}{$\begin{array}{l}\text { Diámetros } \\
\qquad(\mathrm{cm})\end{array}$} & $\mathrm{DE}$ & 41.446 & 36.000 & 51.000 & 3.201 \\
\hline & DD & 67.723 & 61.000 & 75.000 & 3.173 \\
\hline & $\mathrm{AL}$ & 35.872 & 30.000 & 41.000 & 2.626 \\
\hline & LOI & 184.191 & 169.000 & 202.000 & 8.818 \\
\hline & DL & 147.936 & 127.000 & 164.000 & 8.181 \\
\hline & All & 11.127 & 8.000 & 14.000 & 1.596 \\
\hline & $A G$ & 47.297 & 39.000 & 57.000 & 3.380 \\
\hline & $L G$ & 51.085 & 44.000 & 58.000 & 3.315 \\
\hline & AnC & 49.127 & 39.000 & 57.000 & 4.025 \\
\hline \multirow{2}{*}{$\begin{array}{l}\text { Perímetros } \\
\text { (cm) }\end{array}$} & PT & 175.212 & 152.000 & 199.000 & 8.936 \\
\hline & PC & 16.595 & 14.000 & 19.000 & 0.876 \\
\hline
\end{tabular}


Como se define en la literatura "una agrupación o raza en la que se encuentra que todas las variables están significativamente correlacionadas, es una raza que corresponde a un modelo armónico; medianamente armónico cuando el número de correlaciones significativas entre las diversas variables ronde el $50 \%$ y cuando solo están correlacionadas el $25 \%$ de las variables, se puede decir que tiene un modelo poco armónico", (Martínez, 1995).

Tabla 2. Matriz de correlaciones de las 18 variables estudiadas en las hembras de raza Blanco Orejinegro (BON).

\begin{tabular}{|c|c|c|c|c|c|c|c|c|c|c|c|c|c|c|c|c|c|c|}
\hline & & & & & & & & $M$ & $2 \mathrm{D}$ & RRELACI & IONES & $\overline{\mathrm{VAL}}$ & $\overline{E E r}$ & valc & & & & \\
\hline & Acz & $\mathrm{LCz}$ & $\mathrm{LF}$ & LC & $\mathrm{AC}$ & DB & $\mathrm{DE}$ & DD & PT & PC & LOI & $\mathrm{DL}$ & AEG & $\mathbf{A G}$ & LG & AnC & All & $\overline{A L}$ \\
\hline Acz & &, 5565 & 0,41 & \begin{tabular}{l|l|}
14 & 0,135 \\
\end{tabular} & 0,2501 & 000 & 0,521 & & & & 0,4103 & $0,00<1$ & $0,0<03$ & 0,6776 & , & & & 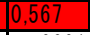 \\
\hline & & $p=, 000$ & $11 \mathrm{p}=, 0 \mathrm{C}$ & $041 p=, 3654$ & $\mathrm{p}=, 0900 \mathrm{p}=$ & $p=, 0001$ & $1 \mathrm{p}=, 0001$ & $p=, 0001$ & $p=, 0001$ & $p=, 0001$ & $p=, 0042$ & $2 p=, 0063$ & $p=: 0256$ & $6 \mathrm{p}=, 000$ & $p=, 000$ & $p=, 000$ & $=, 0$ & \\
\hline $\mathrm{LCz}$ & & 1 & $\longdiv { 8 0 t }$ & 0,1834 & 157 & $\overline{0,5546}$ & 0,3575 & 0,5536 & 0,5044 & 0,3544 & 0,4709 & 0,5272 & 0,4383 & 0,5984 & 0,6611 & 0,4762 & 0,428 & 0,57 \\
\hline & & & $p=, 00$ & $001 p=, 2171 \mid p$ & $p=, 0306 \mid p=$ & $p=, 0001$ & $1 p=, 0136$ & $p=, 0001$ & $p=, 0003$ & $p=, 0145$ & $p=, 0008$ & $8 p=, 0001$ & $p=, 0021$ & $p=, 0001$ & $p=, 0001$ & $p=, 0007$ & $p=, 0027$ & $p=, 0001$ \\
\hline$\overline{L R}$ & & & & $-0,2834$ & 0,31180 & 0,3725 & 0,3118 & 0,316 & 0,3221 & 0,3103 & 0,3682 & 0,3353 & 0,3643 & 0,503 & 0,4847 & 0,3506 & 0,4426 & 0,42 \\
\hline & & & & $p=, 0536$ & $\mathrm{p}=, 0329 \mathrm{p}=$ & $p=, 0099$ & $\begin{array}{l}9 \mathrm{p}=, 0328 \\
\end{array}$ & $p=, 0305$ & $p=, 0272$ & $p=, 0338$ & $p=, 0109$ & $9 p=, 0212$ & $p=, 0118$ & $8 \mathrm{p}=, 0003$ & $p=, 0006$ & $p=, 0157$ & $p=, 0018$ & $p=, 0030$ \\
\hline LC & & & & & \begin{tabular}{|l|l}
$-0,0262$ & 0 \\
\end{tabular} & 0,1144 & \begin{tabular}{|l|l|} 
& $-0,0179$ \\
\end{tabular} & 0,2175 & 0,165 & 0,0738 & 0,1369 & 0,1877 & 0,0262 & 0,038 & 0,1322 & 0,0852 & $-0,1469$ & 0,0991 \\
\hline & & & & & $p=, 8612 p=$ & $p=, 4437$ & $87 \mathrm{p}=, 9049$ & $\mathrm{p}=, 1419$ & $p=, 2676$ & $p=, 6216$ & $\mathrm{p}=, 3585$ & $p=, 2063$ & $p=, 8611$ & $p=, 7995$ & $p=3756$ & $\mathrm{p}=, 568$ & $\mathrm{p}=, 3242$ & $p=5073$ \\
\hline$\overline{A C}$ & & & & & 1 & 0,581 & 0,5086 & 0,5696 & 0,6421 & 0,4884 & 0,5188 & 0,5693 & 0,7909 & 0,5776 & 0,6441 & 0,5 & 0,4409 & 0,5 \\
\hline & & & & & & $p=, 0001$ & $1 \mathrm{p}=, 0003$ & $p=, 0001$ & $p=, 0001$ & $p=, 0005$ & $p=, 0002$ & $2 p=, 0001$ & $p=, 0001$ & $1 \mathrm{p}=, 0001$ & $p=, 0001$ & $p=, 0001$ & $\mathrm{p}=, 0019$ & $p=, 0003$ \\
\hline $\mathrm{DB}$ & & & & & + & & 0,6511 & 0,9295 & 0,8927 & 0,5016 & 0,6334 & 0,6482 & 0,5782 & 0,6575 & 0,7129 & 0,7068 & 0,3726 & 0,7131 \\
\hline & & & & & & & $p=, 0001$ & $p=, 0001$ & $p=, 0001$ & $p=, 0003$ & $p=, 0001$ & $p=, 0001$ & $p=, 0001$ & $1 \mathrm{p}=, 0001$ & $p=, 0001$ & $p=, 0001$ & $\mathrm{p}=, 0099$ & $p=, 0001$ \\
\hline $\mathrm{DE}$ & & & & & & & 1 & 0,6242 & 0,7183 & 0,4996 & $\overline{0,4419}$ & 0,4567 & 0,4219 & 0,6039 & 0,5779 & & 884 & \\
\hline & & & & & & & & $p=, 0001$ & $p=, 0001$ & $p=, 0003$ & $p=, 0019$ & $9 p=, 0013$ & $p=, 0031$ & $1 p=, 0001$ & $p=, 0001$ & $p=, 000$ & $p=, 000$ & $p=, 0$ \\
\hline$\overline{D D}$ & & & & & & & & & 0,911 & 0,506 & 0,6939 & 0,6724 & 0,5965 & 0,652 & 0,7439 & 0,7583 & 0,3503 & 0,70 \\
\hline & & & & & & & & & $p=, 0001$ & $p=, 0003$ & $p=, 0001$ & $p=, 0001$ & $p=, 0001$ & $1 \mathrm{p}=, 0001$ & $p=, 0001$ & $p=, 0001$ & $p=, 0158$ & $p=, 0001$ \\
\hline PT & & & & 88 & & & & & & 0,5553 & 0,691 & 0,61 & 0,592 & 0,6971 & 0,7367 & 0,7172 & 0,4186 & 0,657 \\
\hline & & & & o. & & & & & & $p=, 0001$ & $p=, 0001$ & $\mathrm{p}=, 0001$ & $p=, 0001$ & $p=, 0001$ & $p=, 0001$ & $p=, 0001$ & $p=, 0034$ & $p=, 0001$ \\
\hline$P C$ & & & & & & & & & & 1 & 0,4181 & 0,4451 & 0,583 & & & & 年 & 0,44 \\
\hline & & & & & & & & & & & $p=, 0034$ & $4 p=, 0017$ & $p=.000$ & $1 \mathrm{p}=, 0001$ & $p=, 0003$ & $p=, 0005$ & $p=, 0019$ & $p=, 0020$ \\
\hline LOI & & & & & & & & & & & 1 & 0,6094 & 0.4881 & 0,644 & 0,6901 & 0,6999 & 0,4676 & 0,65 \\
\hline & & & & & & & & & & & & $p=, 0001$ & $p=, 0005$ & $p=, 0001$ & $p=, 0001$ & $p=, 000$ & $p=, 0009$ & $p=, 0001$ \\
\hline $\mathrm{DL}$ & & & & & & & $\overline{\overline{\mathrm{N}^{\mathrm{a}}}}$ & & & & & $t_{2}$ & 0,7626 & 0,5406 & 0,7503 & 0,7244 & 0,3451 & \\
\hline & & & ariable & $\begin{array}{l}\text { Correlaciones } \\
\text { Significtivat }\end{array}$ & Variable & Co & $\begin{array}{l}\text { iorrelaciones } \\
\text { ionificativas }\end{array}$ & & & & & & $p=, 0001$ & $\mathrm{p}=, 0001$ & $p=, 0001$ & $p=, 0001$ & $p=, 017$ & $p=, 0001$ \\
\hline AEG & & & $\overline{\mathrm{ACz}}$ & $\frac{\text { Significativas }}{15}$ & $\mathbf{P C}$ & & $\begin{array}{l}\text { ignificativas } \\
16\end{array}$ & & & & & & 1 & 0,62 & & & & \\
\hline & & & $\mathrm{LCz}$ & 16 & LOI & & 16 & & & & & & & $p=, 0001$ & $p=, 0001$ & $p=, 0001$ & $p=, 0057$ & $p=, 0001$ \\
\hline $\mathrm{AG}$ & & & LR & 16 & DL & & 16 & & & & & & & 1 & 7404 & 0,6872 & 62 & 0,7 \\
\hline & & & LC & 0 & AEG & & 16 & & & & & & & & $p=, 0001$ & $p=, 0001$ & $p=, 0001$ & $p=, 0001$ \\
\hline LG & & & $\mathrm{AC}$ & 15 & $\mathbf{A G}$ & & 16 & & & & & & & & 1 & 0,843 & 0,5523 & 0,8302 \\
\hline & & & DB & 16 & LG & & 16 & & & & & & & & & $p=, 0001$ & $p=, 0001$ & $p=, 0001$ \\
\hline AnC & & & DE & 16 & AnC & & 16 & & & & & & & & & 1 & & Ju, \\
\hline & & & $\begin{array}{l}\text { DD } \\
\text { PT }\end{array}$ & $\begin{array}{l}16 \\
16\end{array}$ & $\frac{\text { AII }}{\text { AL }}$ & & $\begin{array}{l}16 \\
16\end{array}$ & & & & & & & & & & $p=, 0001$ & $p=, 0001$ \\
\hline All & & & & & & & & & & & & & & & & & 11 & 0,6417 \\
\hline & & & & & & & & & & & & & & & & & & $p=, 0001$ \\
\hline$A L$ & & & & & & & & & & & & & & & & & & 1 \\
\hline
\end{tabular}

Realizando un análisis de cada uno de los valores de la matriz, se denota que la variable longitud de cráneo fue la única medida que no tuvo ninguna correlación significativa con el resto de las variables. Se observó, que la mayoría de medidas tomadas están positivamente correlacionadas entre sí, permitiendo deducir el 
comportamiento de una variable al tener el conocimiento de una de estas variables correlacionadas en la Tabla 2, además en algún momento definen con mayor precisión la talla y funcionalidad del animal. Por ejemplo, la alzada a la cruz (AC), presentó un valor significativo de correlación $(p<0,01)$, con el perímetro del tórax y se sabe que este ha sido utilizado a través del uso de cintas métricas para determinar el peso corporal de un animal; es decir que, si se carece de la cinta métrica, se podría determinar el peso del animal por su alzada a la cruz.

\section{Análisis de índices morfométricos:}

Los índices morfométricos estudiados se clasificaron en índices raciales y en índices funcionales Índice Cefálico (ICE): anchura de la cabeza/longitud de cabeza. Índice Facial (IFA): anchura de la cara/longitud de cara. Índice Torácico (ITO): diámetro bicostal/diámetro dorso esternal. Índice Corporal (ICO): diámetro longitudinal/perímetro torácico. Índice de Proporcionalidad (IPR): alzada a la cruz/diámetro longitudinal. Índice Pelviano (IPE): anchura de la grupa/longitud de la grupa. Índice Metacarpo-Torácico (IMTOR): perímetro de la caña/perímetro torácico. Índice Metacarpo-Costal (IMCOS): perímetro de la caña/diámetro bicostal. Profundidad Relativa del Tórax (IPRT): diámetro dorso-esternal/alzada a la cruz. Índice Pelviano Transversal (IPETR): anchura de la grupa/alzada a la cruz. Índice Pelviano Longitudinal (IPELO): Iongitud de la grupa/alzada a la cruz. Peso relativo o Índice de Compacidad (PREL): peso corporal/alzada a la cruz. Carga de la caña (PRC): perímetro de la caña/peso corporal. Espesor relativo de la caña (IECÑA): perímetro de la caña/alzada a la cruz (Tabla 3).

Índice Cefálico (ICE): anchura de la cabeza/ longitud de cabeza. Índice Facial (IFA): anchura de la cara/longitud de cara. Índice Torácico (ITO): diámetro bicostal/diámetro dorso esternal. Índice Corporal (ICO): diámetro longitudinal/perímetro torácico. Índice de Proporcionalidad (IPR): alzada a la cruz/diámetro longitudinal. Índice Pelviano (IPE): anchura de la grupa/longitud de la grupa. Índice Metacarpo-Torácico (IMTOR): perímetro de la caña/perímetro 
torácico. Índice Metacarpo-Costal (IMCOS): perímetro de la caña/diámetro bicostal. Profundidad Relativa del Tórax (IPRT): diámetro dorso-esternal/alzada a la cruz. Índice Pelviano Transversal (IPETR): anchura de la grupa/alzada a la cruz. Índice Pelviano Longitudinal (IPELO): Iongitud de la grupa/alzada a la cruz. Peso relativo o Índice de Compacidad (PREL): peso corporal/alzada a la cruz. Carga de la caña (PRC): perímetro de la caña/peso corporal. Espesor relativo de la caña (IECÑA): perímetro de la caña/alzada a la cruz.

Tabla 3. Valores medios, mínimos, máximos y desviación estándar del peso corporal y de los 14 índices morfométricos analizados en hembras BON

\begin{tabular}{ccccc}
\hline Variable (cm) & Media & Mínimo & Máximo & Desv. Std \\
\hline PESO & 439.042 & 280.000 & 640.000 & 68.612 \\
ICE & 46.720 & 41.180 & 54.550 & 2.474 \\
IFA & 75.829 & 61.760 & 100.000 & 6.651 \\
ITO & 100.587 & 95.160 & 105.8000 & 2.430 \\
ICO & 84.501 & 76.970 & 91.720 & 3.967 \\
IPR & 83.152 & 74.390 & 91.730 & 3.816 \\
IPE & 92.662 & 83.020 & 106.820 & 4.695 \\
IMTOR & 9.481 & 8.540 & 10.630 & 0.461 \\
MICOS & 24.401 & 21.920 & 27.870 & 1.394 \\
IPRT & 55.178 & 50.000 & 60.500 & 2.166 \\
IPETR & 38.516 & 33.880 & 43.090 & 2.267 \\
IPELO & 41.601 & 36.070 & 45.450 & 2.106 \\
PREL & 356.872 & 241.380 & 481.200 & 48.931 \\
PRC & 3.854 & 2.660 & 5.310 & 0.525 \\
IECÑA & 13.520 & 12.070 & 14.840 & 0.632 \\
\hline
\end{tabular}

Índices raciales. (ICO): $84,5 \pm 3.9 \mathrm{~cm}$ Indica que los animales muestreados son de troncos acortados, anchos y espesos en relación con sus capacidades torácicas, animales zootecnicamente definidos como Brevilineos (ITO): $100 \pm 2,4$ $\mathrm{cm}$ relaciona las medidas de diámetro bicostal con diámetro dorsoestrenal, nos 
indica que son animales ultrabrevilineos. Este índice presenta variación, ya que su valor está afectado por el diámetro bicostal que es una medida que normalmente tiene gran variabilidad, teniendo en cuenta que depende del estado de carnes y reproductivo del animal al momento de tomar la medida. Las vacas BON exceden casi en el doble al ITO de las vacas CC el cual fue de 55,04 \pm 12,83 cm., (Sastre, 2003), poniéndose en evidencia la amplitud de la cavidad torácica y por tanto pulmonar de las BON, característica adquirida como adaptación a las alturas de las zonas montañosas en las que se encuentran, diferente a las CC las cuales viven en zonas bajas.

Índices funcionales. (IMCOS): 24,4 $\pm 1,3 \mathrm{~cm}$, indica mayor finura del esqueleto y mayor amplitud de la cavidad torácica; ya que a mayor diámetro bicostal, menor será el IMCOS. No se encontraron estudios en la revisión de literatura con otras razas criollas, para compararlos con los de este trabajo. (IPRT): 55,1 2,1 cm. (IPE): 92,6 \pm 4,6 cm. (PRC): $3,8 \pm 0,5 \mathrm{~cm}$. (IECÑA): 13,5 $\pm 0,6 \mathrm{~cm}$. (IMTOR): 9,4 \pm 0,4 cm, según Aparicio (1960), en vacas de aptitud láctea, el perímetro de la caña (16,5 cm.) debe estar comprendido diez veces en el perímetro torácico $(175,2 \mathrm{~cm}$.); siendo, por tanto, el índice más favorable cuanto más se acerque a la cifra especificada como base. (IPETR): $38,5 \pm 2,2 \mathrm{~cm}$, relaciona la alzada a la cruz con el diámetro dorsoesternal, indica que son animales de gran amplitud de la cavidad torácica en comparación con sus extremidades acortadas, las cuales se consideran apropiadas para el desplazamiento de zonas montañosas y terrenos abruptos, además indica que son animales especializados en la producción de carne ya que el diámetro dorsoesternal $(67,7)$ es superior a la mitad de la alzada a la cruz (61). (IPELO):41,6 $\pm 2,1 \mathrm{~cm}$. (PREL): $356,8 \pm 48,9 \mathrm{~cm}$. (IPR): 83,1 $\pm 3,8 \mathrm{~cm}$ (Aranguren, 2001).

Las anteriores medidas de la caja pélvica denotan una buena amplitud de caderas, buena proporcionalidad de los diámetros pélvicos con respecto a la conformación general, por lo que raras veces ocurran distocias en estos animales. Luego de analizar en general las variables; se observó, que algunas características tales 
como profundidad, perímetro oblicuo del tórax, ancho del pecho, diámetro longitudinal, alzadas, dan a conocer que son animales rectangulares cilíndricos, característica propia de la línea cárnica (Pere-Miquel, 2007).

\section{CONCLUSIONES}

- El modelo morfoestructural del ganado Blanco Orejinegro (BON), en este estudio, demostró que la raza es armónica; indicando que existe, en el hato estudiado, un alto grado de homogeneidad que podría atribuirse al hecho de ser un hato cerrado a la influencia de poblaciones exógenas con otras tendencias genéticas.

- El desnivel entre las alturas de la cruz y anca o grupa, donde la primera es menor, y la fortaleza de sus extremidades anteriores, así como el valor de índice corporal y su interpretación de animales de troncos acortados, anchos y espesos, son indicativos claros de su adaptación a terrenos abruptos y aptitud para el trabajo y producción de carne.

- La talla del ganado BON, en este estudio, comparada con otras razas criollas como Hartón del Valle, Sanmartinero, Casanareño y Caqueteño es inferior en aproximadamente $10 \mathrm{~cm}$, y ese menor tamaño y las características antes descritas son una corroboración adicional que su talla obedece a su adaptación a la zona abrupta de los Andes colombianos, en dónde animales de gran talla difícilmente se podrían desplazar.

- Los resultados de este estudio son una contribución a la caracterización de la raza BON y deben servir de base para realizar otros, que analicen las relaciones existentes entre las medidas e índices morfométricos con peso, características reproductivas y aptitudes productivas de carne, leche y trabajo de esta y otras razas. 


\section{RECOMENDACIONES}

- Este trabajo puede servir como base para la realización de otros estudios y para la elaboración de programas de selección tendientes a corregir aquellas características que se consideren inapropiadas para el desarrollo futuro de las razas criollas.

- Este tipo de estudio debe complementarse con el registro sistemático de las características de importancia económica como la reproducción, crecimiento y producción de leche.

\section{REFERENCIAS BIBLIOGRÁFICAS}

1. Aparicio, S. G. Exterior de los grandes animales domésticos, Morfología externa, Córdoba, (España), ed. Imprenta Moderna, p. 197-238. 1960.

2. Aranguren, F. J. Introducción general a la clasificación morfológica de los bovinos de carne con referencia a la raza pirenaica. Revista Federación Española de Asociaciones de Ganado Selecto (FEAGAS), No 19. Madrid (España). p 50-69. 2001.

3. Buitrago, F; Gutiérrez, I. D. Censo y caracterización de los sistemas de producción del ganado criollo y colombiano. Fondo Nacional del Ganado (FEDEGAN); German Martínez C (ed.), p 65-73. 1999.

4. Floriano, P. L.; Trujillo, Y. Caracterización faneróptica, morfológica y morfométrica del ganado criollo Caqueteño. Tesis de grado, Universidad de la Amazonía. 2005.

5. Gómez, A. M. Influencia de tamaño en eficiencia reproductiva y productiva de vacas Brahman en el trópico. Universidad de la Salle. Facultad de Medicina Veterinaria. Bogotá D. C. 1998.

6. Herrera, M., Rodero, E., Gutiérrez, M. J., Peña, F., Y Rodero, J. M. Aplication of multifactorial discriminant análisis in the morphostructural differentiation of andalusian caprine breeds. Small Rumiant Reserch, p 39-47. 2003.

7. Husu K. J. El bienestar animal en la legislación de la unión europea y a escala internacional. En: Actas del seminario: La Institucionalización del bienestar animal, un requisito para su desarrollo normativo, científico y productivo, Santiago de Chile, 11-12 de noviembre de 2004.

8. López A., Saldarriaga O., Arango A., Rúgeles M., Ossa J, Zuluaga F., Olivera M., Bermúdez N., Bedoya G., Ossa J. Ganado Blanco Orejinegro (BON): Una alternativa para la producción. Rev Col Cienc Pec., 14: 2. 2001.

9. Martínez, C. G. El ganado criollo colombiano Blanco Orejinegro (BON). Animal Genetic Resources Information. UNEP FAO. (Roma) 9: 33-44. 1992. 
10. Martinez, C. G. The Colombian cattle breed. Proceedings of the Third Global Conference on Conservation of Domestic Animal Genetic Resources. En: R.D. Crawford, E.E. Lister, y J.T. Buckley (Ed.). Rare Breed International. Kingston. Ontario, Canadá, p 161-166. 1995.

11. Martínez, C. G. Censo y caracterización de los sistemas de producción del ganado criollo y colombiano. En: Memorias. Censo y caracterización de los sistemas de producción del ganado criollo y colombiano. Bogotá, D.C. Colombia. p 13-64. 1999.

12. Ministerio de Agricultura y Desarrollo Rural (MADR). Situación de los recursos Zoogenéticos en Colombia. Bogotá D.C. 2003.

13. Pere-Miquel I. Índices de interés funcional en la raza bovina "Bruna Dels Pirineus" REDVET. Revista Electrónica de Veterinaria, VIII: 6: 1-7. 2007.

14. Pinzón M. E. Historia de la ganadería bovina en Colombia. Banco Ganadero (Col.) 4 (1): 208. 1984.

15. S.A.S. SAS user's guide: Statistics. Statistical Analysis System. Inc., Cary, N. C. 1995.

16. Sastre, H. J. Ganado criollo Casanare, descripción etnológica y estrategias para su rescate y conservación. España; Departamento de producción animal universidad de Córdoba. 2003.

17. Sierra L. E.; Velásquez P. D. Parámetros reproductivos y algunas medidas bovinométricas del ganado criollo Sanmartinero en la granja Iracá (San Martín - Meta). Tesis de grado para optar el título de Médico Veterinario y Zootecnista. Universidad de Los Llanos. Villavicencio. 2000.

18. Vaca M. E. Análisis retrospectivo del comportamiento reproductivo y crecimiento predestete de un hato mixto de ganado criollo Sanmartinero y Cebú y sus cruces recíprocos. Trabajo de Grado en Zootecnia. Facultad de Ciencias Agropecuarias, Fundación Universitaria Agraria De Colombia UNIAGRARIA". Bogotá. 2002.

19. Valderrama M. Razas Criollas y colombianas puras. Memorias. Convenio 13501. Asocriollo. 2003.

20. Warriss P. the transport of animals: a long way to go. The Veterinary Journal 168: 213-214, 2004. 